\title{
Role of Endothelial Lipase in Plasma HDL levels in a Murine Model of Hypertriglyceridemia
}

\author{
Hanayo Tanaka ${ }^{1}$, Tatsuro Ishida ${ }^{1}$, Thomas P. Johnston ${ }^{2}$, Tomoyuki Yasuda ${ }^{1}$, Tomomi Ueyama ${ }^{1}$, \\ Yoko Kojima ${ }^{1}$, Ramendra K, Kundu ${ }^{3}$, Thomas Quertermous ${ }^{3}$, Yuichi Ishikawa ${ }^{4}$, and Ken-ichi Hirata ${ }^{1}$ \\ ${ }^{1}$ Division of Cardiovascular Medicine, Kobe University Graduate School of Medicine, Kobe, Japan \\ ${ }^{2}$ Division of Pharmaceutical Sciences, School of Pharmacy, University of Missouri-Kansas, Kansas City Missouri, USA \\ ${ }^{3}$ Division of Cardiovascular Medicine, Stanford University School of Medicine, Stanford, California, USA \\ ${ }^{4}$ Kobe University Graduate School of Health Sciences, Kobe, Japan
}

\begin{abstract}
Aim: Hypertriglyceridemia is the most common cause of low plasma high-density lipoprotein cholesterol (HDL-C) levels; however, the correlation between high triglyceride (TG) and low HDL-C remains unclear. Endothelial lipase (EL) is a determinant of plasma HDL levels. We investigated the role of EL in HDL metabolism in a murine model of acute hypertriglyceridemia.

Methods and Results: To establish TG-dominant hyperlipidemia, EL-/- and wild-type (WT) mice were injected with Poloxamer-407 (P-407, $0.5 \mathrm{~g} / \mathrm{kg}$, i.p.). A single injection of P-407 resulted in a marked increase in plasma TG and cholesterol levels together with a decrease in HDL-C levels. Although plasma TG levels were similar in $E L-/-$ and WT mice after P-407 injection, HDL-C levels were $80 \%$ higher and the HDL particle size was significantly larger in $E L-/-$ mice than in WT mice. P-407 treatment inhibited plasma lipoprotein lipase activity and EL phospholipase activity, without decreasing their expressions. Adenovirus-mediated overexpression of EL in the liver reduced plasma HDL-C levels in both normo- and hyperlipidemic mice, while overexpression of catalytically inactive EL reduced HDL-C levels in hyperlipidemic mice. Cell culture experiments revealed that both catalytically active and inactive EL promoted cellular HDL uptake to the same extent.

Conclusion: EL regulates plasma HDL levels in mice in the normolipidemic as well as the acute hypertriglyceridemic state. EL can modulate plasma HDL-CHOL levels through both its lipolytic and ligand-binding functions in hypertriglyceridemic mice, while lipolytic activity appears to be the main determinant for its effects on HDL metabolism in normolipidemic mice.
\end{abstract}

J Atheroscler Thromb, 2009; 16:327-338.

Key words; Triglyceride, Cholesterol, High-density lipoproteins, Endothelial lipase

\section{Introduction}

There is growing recognition that metabolic syndrome is an alarmingly major worldwide public health problem, because it is arguably now the major cause of cardiovascular diseases and premature death ${ }^{1)}$. Characteristics of metabolic syndrome include abdom-

Address for correspondence: Tatsuro Ishida, Division of Cardiovascular Medicine, Kobe University Graduate School of Medicine, 7-5-1 Kusunoki-cho, Chuo-ku, Kobe 650-0017, Japan

E-mail: ishida@med.kobe-u.ac.jp

Received: October 15, 2008

Accepted for publication: January 6, 2009 inal obesity, atherogenic dyslipidemia, increased blood pressure, insulin resistance, and pro-thrombotic and pro-inflammatory states ${ }^{1)}$. As for dyslipidemia, elevated plasma triglyceride (TG) levels and low plasma concentrations of high-density lipoprotein (HDL)cholesterol (CHOL) have emerged as diagnostic criteria. Patients with hypertriglyceridemia frequently have low plasma levels of HDL-CHOL, and these lipid disorders synergistically contribute to an increased risk of coronary heart disease ${ }^{2}$. Moreover, hypertriglyceridemia, low HDL-CHOL, and qualitative changes in low-density lipoprotein (LDL) account for the typical dyslipidemia in insulin-resistant stages ${ }^{3)}$. There is thus great interest in determining the mechanisms respon- 
sible for reduced HDL levels in hypertriglyceridemia associated with metabolic syndrome and insulin resistance.

Previous studies have indicated that hypertriglyceridemia per se can be a cause of low HDL levels ${ }^{2,4)}$. In addition, HDL-CHOL levels in hypertriglyceridemic patients are regulated by cholesteryl ester transfer protein (CETP), lecithin:cholesterol acyltransferase (LCAT), hepatic TG lipase (HL), and lipoprotein lipase $(\mathrm{LPL})^{5)}$; however, these plasma factors can account for only half of the variations in plasma concentrations of HDL-CHOL in hypertriglyceridemic patients ${ }^{5)}$, and indeed, it has been postulated that variations in HDLCHOL levels in humans are approximately 50\% genetically determined ${ }^{6}$. Endothelial lipase (EL) is one of these genetic determinants of plasma HDL levels ${ }^{7,8)}$. Previous animal studies have documented an inverse correlation between EL expression and plasma HDLCHOL levels ${ }^{8-10)}$. Association-based human genetic studies have shown that variations in the EL genomic $L I P G$ locus are linked to differences in circulating HDL-CHOL levels ${ }^{10)}$. Plasma EL levels have been shown to be associated with plasma HDL levels in patients with metabolic syndrome ${ }^{11)}$. These lines of evidence imply that EL may play a role in the genesis of low HDL accompanied by hypertriglyceridemia or insulin resistance in metabolic syndrome.

Poloxamer 407 (P-407; Pluronic F-127 ${ }^{\circledR}$ ) is a block copolymer consisting of repeating polyoxylene and polyoxypropylene units and is categorized as a nonionic surfactant polyol. It has been shown to produce a profound hypertriglyceridemia in rodents ${ }^{12,13)}$. A single injection of P-407 $(0.25-1.0 \mathrm{~g} / \mathrm{kg}$, i.p.) in mice produced a 10 -fold increase in plasma $\mathrm{CHOL}$ that peaked at 24 hours and had returned to control levels by 96 hours following treatment ${ }^{14)}$, while plasma TG levels changed even more markedly than $\mathrm{CHOL}$ levels following the administration of P-407 ${ }^{14)}$. These effects are primarily believed to be the result of LPL and HL inhibition ${ }^{12)}$. Thus, P-407-induced TG-dominant hyperlipidemia resembles Type IV hypercholesterolemia in humans ${ }^{12}$. In this study, we aimed to explore the effect of P-407 on EL expression and function, and to identify the role of EL in HDL metabolism in a P-407-induced murine model of acute hypertriglyceridemia.

\section{Materials and Methods}

\section{Animal Preparation}

All animal experiments were conducted at Kobe University Graduate School of Medicine according to the institutional Guidelines for Animal Experi- ments. Male homozygous $E L-/-$ mice, which had been backcrossed with the C57BL6/J strain for $>13$ generations ${ }^{9)}, 8-12$ weeks of age, and age-matched C57BL6/J wild-type (WT) mice (Crea Japan, Inc., Tokyo, Japan) were used in this study. The mice were maintained on a chow diet containing $4.6 \%$ fat, $24.3 \%$ protein, $3.7 \%$ dietary fiber, and $6.7 \%$ ash (Crea Rodent Diet CE-2, Crea Japan). EL-/- was genotyped by polymerase chain reaction (PCR) using the following primers: 5 '-cctccttcatcacgcttaggtgtct-3' (forward primer corresponding to the first intron in the LIPG genomic sequence), 5'-cacctacctcaagtcacccttcaag-3' (reverse primer corresponding to the first intron in the LIPG), 5'-gtggatgtggaatgtgtgcgaggcc-3' (forward primer corresponding to the PGK promoter in the $\mathrm{pKO}$ Scrambler NTKV1901 targeting vector). PCR was performed for 35 cycles at $93^{\circ} \mathrm{C} 1 \mathrm{~min}, 54^{\circ} \mathrm{C} 2 \mathrm{~min}$, and $72^{\circ} \mathrm{C} 2 \mathrm{~min}$, and yielded a 236-bp in single band $E L-/-$ mice, a 436-bp single band in $E L+/+$ mice, and 236- and 436-bp double bands in $E L+/-$ mice. Mice were injected with P-407 (0.5 g/kg, i.p.; Molecular Probes, Eugene, OR) after overnight fasting.

\section{Analysis of Plasma Lipids}

For lipid analysis, whole blood was collected by retro-orbital bleeding or cardiac puncture following an overnight fast. Plasma was collected by centrifugation at $8,000 \mathrm{~g}$ for $5 \mathrm{~min}$ at $4{ }^{\circ} \mathrm{C}$. Total $\mathrm{CHOL}, \mathrm{TG}$, and phospholipid (PL) levels were measured using commercially available kits (Wako Pure Chemicals, Tokyo, Japan). For separation of plasma lipoproteins by ultracentrifugation, $1.0 \mathrm{~mL}$ pooled plasma from 2-3 male mice was used, and CHOL, TG, and PL levels in each lipoprotein fraction were determined by biochemical assays ${ }^{15,16)}$. Furthermore, plasma lipoprotein profiles and sizes were analyzed with an on-line dual enzymatic method for simultaneous quantification of CHOL and TG by HPLC (LipoSEARCH ${ }^{\circledR}$ ) from Skylight Biotech, Inc. (Akita, Japan) according to the specified procedure ${ }^{17,18)}$. Briefly, $200 \mu \mathrm{L}$ of $20 \mathrm{x}$ salinediluted sera was injected into two tandem connected TSK gel Lipopropak XL columns $(300 \times 7.8 \mathrm{~mm}$; Tosoh, Tokyo, Japan), and CHOL and TG contents in lipoproteins separated by size were determined using enzymatic reagents specially prepared by Kyowa Medex (Tokyo, Japan). Total CHOL and TG concentrations were calculated by comparing the total area under the chromatographic curves of a calibration standard of known concentration ${ }^{18)}$.

\section{Adenovirus-Mediated EL Gene Transfer}

Wild-type human EL, catalytically inactive human EL, in which the alanine (EL-S149A) was 
substituted for the active site serine, or LacZ were expressed by gene transfer. Recombinant adenoviruses encoding human EL (AdEL), human EL-S149A (AdELS149A), and LacZ (AdLacZ, a negative control), were constructed as described previously ${ }^{19)}$. Mice were intravenously injected via the tail vein with $1 \times 10^{11}$ particles of AdEL, AdEL-S149A, or AdLacZ ${ }^{19}$. Fortyeight hours after the adenoviral injection, the mice were injected with P-407, and 16 hours later, blood was drawn for lipoprotein analysis.

\section{Determination of Cellular Uptake of HDL}

HDL ( $\mathrm{d}=1.063$ to $1.215 \mathrm{~g} / \mathrm{mL}$ ) was isolated from healthy human plasma by sequential ultracentrifugation, dialyzed against PBS/EDTA, and labeled with the fluorescent probe 1,1'-dioctadecyl-3, 3,3', 3'-tetramethylindocarbocyanide perchlorate (DiI; Molecular Probes). HDL was incubated for 8 hours at $37^{\circ} \mathrm{C}$ with $50 \mu \mathrm{L}$ DiI in DMSO $(3 \mathrm{mg} / \mathrm{mL})$ for each milligram of protein. The labeled HDL was then re-isolated by ultracentrifugation and stored under $\mathrm{N}_{2}$ gas.

Confluent monolayers of COS-7 cells were infected with a $1 \times 10^{8}$ multiplicity of infection (MOI) of AdLacZ, AdEL-WT, or AdEL-S149A for 24 hours, followed by incubation for 2 hours with $5 \mu \mathrm{L}$ cholesterol $/ \mathrm{mL}$ of DiI-labeled HDL in combination with a 50-fold excess of unlabeled HDL. Cells were washed twice with PBS containing $0.2 \%$ bovine serum albumin, and then twice with PBS only. Fluorescence intensity was measured on a FACScan (BD Biosciences, Franklin Lakes, NY), with excitation and emission wavelengths set at $520 \mathrm{~nm}$ and $580 \mathrm{~nm}$. The results are expressed as the mean fluorescence intensity (MFI) and as a percentage of the MFI of AdLacZinfected COS-7 cells. In some experiments, fluorescence intensity was measured with a laser-scanning confocal imaging system (BZ-8000; Keyence, Osaka, Japan). To confirm the level of transgene expression, $10 \mathrm{U} / \mathrm{mL}$ heparin was added to the culture medium, and EL expression in culture medium was evaluated by Western blotting.

\section{Lipase Expression Analysis}

Expressions of EL, HL, and LPL in mouse tissues or cultured cells were analyzed with RNase protection assays or real-time PCR, or with Western blotting utilizing an anti-human EL monoclonal antibody9). For RNase protection assays, cDNA fragments of EL (coding from +1 to +281 ), LPL (coding from +164 to +395 ), and HL (coding from +237 to +410 ) cDNA were obtained with PCR using the following primers. Mouse EL; 5'-gatgcgaaacacggtttt-3', and 5'-tcgtccatccatgaatgatg-3'. Mouse LPL; 5'-tagttccagcagcaaagcaga- aggg-3' and 5'-tagacacagagtctgctaatccagg-3'. Mouse HL; 5'-gaggccagagacatgggaaatcccc-3' and 5'-gaagagcaggaatctggtctctggc- 3 '. PCR products were cloned into the pGEM T-easy vector (Promega, Madison, WI), and linearized with SpeI. Cyclophilin cDNA template was purchased from Ambion, Inc. (Austin, TX). $\left[{ }^{32} \mathrm{P}\right] \mathrm{UTP}$-labeled antisepses riboprobes were synthesized with T7 RNA polymerase, and the RNase protection assay was performed using an RPAIII kit (Ambion) ${ }^{9}$, 20). Protection of murine EL, LPL, HL, and cyclophilin transcripts resulted in a labeled fragment of 281, 232, 174 and 103 nucleotides, respectively. A Fujix BAS2000 bio-imaging analyzer (Fujifilm, Tokyo, Japan) was used to standardize the relative signal intensity of EL mRNA expression with that of cyclophilin mRNA. For real-time PCR, purified RNA from mouse tissue was reverse-transcribed using an ExScriptTM RT reagent kit (TaKaRa, Shiga, Japan). Quantitative gene expression analysis was performed on an ABI PRISM 7500 Real-Time PCR System (Applied Biosystems, Foster City, CA) using SYBR Green technology. PCR primers were purchased from TaKaRa as follows;

Mouse LPL; 5'-gccgagagcgagaacattcc-3', and 5'tgtccacctccgtgtaaatcaaga-3'.

Mouse HL; 5'-gcccattgcccactatgacttc-3', and 5'atgctctgcaggtcactgtgttg-3'.

Mouse EL; 5'-ggtgagctaaggacccagcaag-3', and 5'cacatcaggaacatctgggtagga- 3 '.

Mouse $\beta$-actin; 5'-ccc taaggccaaccgtgaa- 3 ', and 5'gttgaaggtctcaaacatgatctg-3'.

Human EL; 5'-attagggaccctgaggctttaagtg-3', and 5'-atatctggcaaccggccatc-3'.

Human GAPDH; 5'-acggatttggtcgtattgggc-3', and 5'-ttgacggtgccatggaatttg-3'.

\section{Lipase Assay}

Mouse blood was collected into EDTA-containing tubes before and 10 minutes after intravenous injection of heparin $(150 \mathrm{U} / \mathrm{kg})$. Plasma TG lipase activity was measured with the CONFLUORIP kit (Progen, Heidelberg, Germany). Phospholipase activity was quantified as described previously ${ }^{9,21)}$. In a subset of in vitro experiments, human umbilical vein endothelial cells, or COS7 cells which stably overexpress human $\mathrm{EL}^{7)}$, were incubated with P-407 for 24 hours. The culture medium was collected after the addition of heparin and concentrated 20-40 times for phospholipase assays ${ }^{7}$.

\section{Statistical Analysis}

Data are expressed as the mean \pm SE. Unpaired Studently $t$-test was used to identify significant differ- 
ences between groups. One-way ANOVA was used to determine differences among three or four groups, with Bonferroni's test used for post-hoc analysis. Repeated measures ANOVA was used to compare lipid fraction results obtained from HPLC. $P$ values $<0.05$ were considered significant.

\section{Results}

\section{P-407 Induces Triglyceride-Dominant Hyperlipid- emia}

Mouse plasma lipid profiles were determined with a biochemical assay. Under basal conditions, total CHOL in EL-/- mice approximately doubled compared to that WT mice (Fig. 1A). When P-407 was injected into these mice, the plasma showed marked hyperlipidemia, while the total CHOL level increased up to $12-15 \mathrm{mmol} / \mathrm{L}$ in both WT and $E L-/-$ mice. The CHOL level after P-407 was moderately but significantly higher in $E L-/-$ mice than in WT mice.

Plasma TG levels were low in these fasted mice at baseline (Fig. 1B), but P-407 administration resulted in a marked elevation of plasma TG levels in both mouse groups. That there was no difference in TG levels between mouse groups after P-407 injection (Fig. 1B) suggests that EL deficiency does not augment hypertriglyceridemia in this model.

\section{P-407 Increases VLDL, but Reduces the Level of HDL, which is Restored by EL Deficiency}

The lipoprotein analysis using ultracentrifugation provided direct evidence that the increase in CHOL in EL-/- mice at baseline reflects the 70\% increase in the HDL fraction (Table 1). LDL-CHOL was slightly enhanced in $E L-/-$, but VLDL-CHOL was not affected by EL deficiency. When mice were treated with P-407, VLDL-CHOL primarily increased, but the levels were similar for the two mouse groups. P-407 caused an increase in LDL-CHOL in both groups, with $E L-/-$ mice showing a significantly higher LDL-CHOL level than WT mice. These increases in LDL-CHOL were considered a result of the catalytic action of EL on apoB-containing lipoproteins. In contrast, HDL-CHOL was reduced by $40 \%$ in response to P-407 in both groups. Interestingly, the HDL-CHOL level in EL-I- mice was still $80 \%$ higher than in WT after P-407 treatment, even though plasma TG levels were similar. These findings indicate that complete EL inactivation by gene deletion leads to an increase in the low level of HDLCHOL in P-407-induced hypertriglyceridemia.

VLDL-TG levels were similar in EL-/- and WT mice. When mice were treated with P-407, VLDL-TG
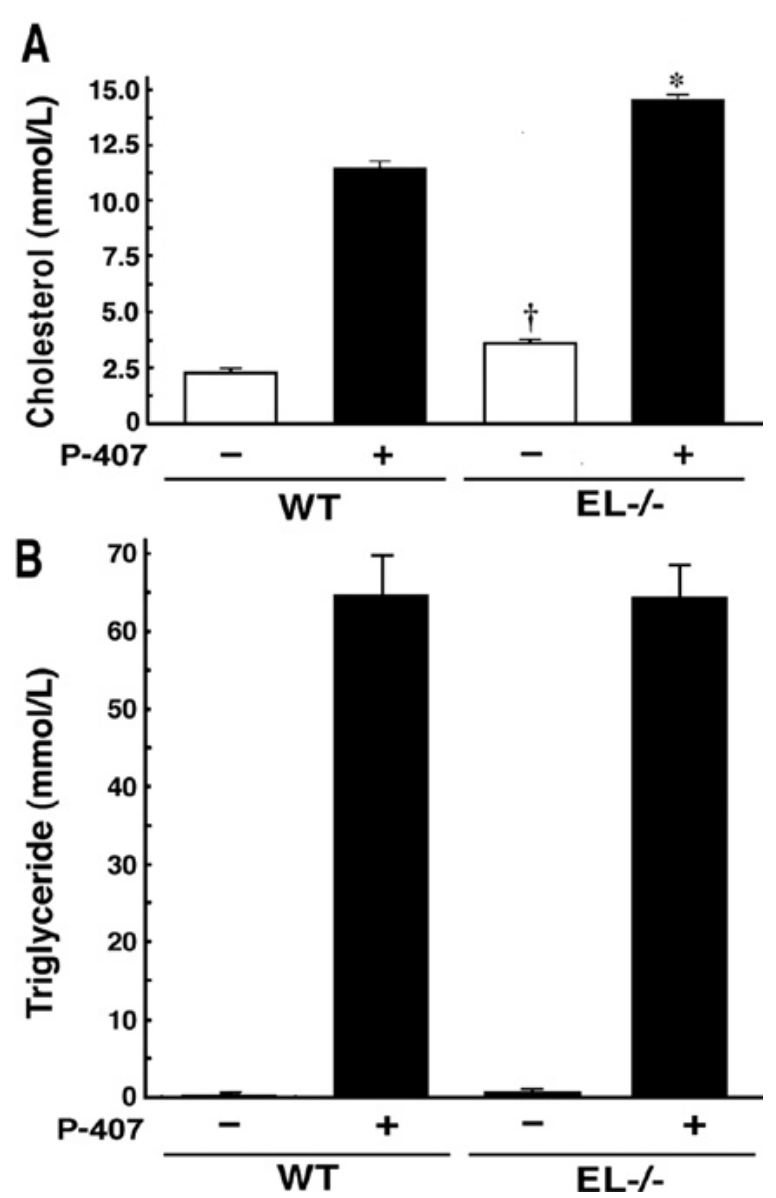

Fig. 1. Plasma cholesterol and triglyceride levels after P-407 treatment.

P-407 was injected into wild-type (WT) and endothelial lipasedeficient $(E L-/-)$ mice, and plasma cholesterol (CHOL) and triglyceride (TG) levels were determined with biochemical assays. Data are expressed as the mean \pm SE. ${ }^{*} p<0.05$ and ${ }^{\dagger} p<0.01$ vs. corresponding WT value ( $n=15$ in each group).

markedly increased, but there was still no significant difference in VLDL-TG between groups (Table 1). At baseline, HDL-TG was slightly higher in $E L-/-$ than in WT mice, and was increased by P-407 without any difference in levels between groups. HDL-PL levels were higher in EL-/- than in WT mice both before and after P-407 injection, reflecting the absence of EL phospholipase activity. LDL-PL also increased in EL-/- mice before and after P-407 injection. VLDL-PL was not affected by EL deficiency.

\section{P-407 Increases HDL-TG and EL Inactivation Increases HDL-CHOL}

HPLC was used for further characterization of the lipoprotein profile (Fig. 2). When mice were treated with P-407, VLDL-CHOL markedly increased 
Table 1. Fasting plasmas lipid and lipoprotein profile in WT and EL-/- mice before and 16 hours after P-407 injection

\begin{tabular}{|c|c|c|c|c|c|c|}
\hline & $\begin{array}{c}\text { WT } \\
-\mathrm{P}-407\end{array}$ & $\begin{array}{l}E L-/- \\
-\mathrm{P}-407\end{array}$ & $p$ value & $\begin{array}{c}\text { WT } \\
+\mathrm{P}-407\end{array}$ & $\begin{array}{l}E L-/- \\
+\mathrm{P}-407\end{array}$ & $p$ value \\
\hline \multicolumn{7}{|c|}{ Cholesterol (mmol/L) } \\
\hline HDL-CHOL & $1.74 \pm 0.05$ & $2.95 \pm 0.08$ & $<0.0001$ & $0.99 \pm 0.03$ & $1.78 \pm 0.16$ & $<0.001$ \\
\hline LDL-CHOL & $0.31 \pm 0.03$ & $0.58 \pm 0.06$ & $<0.05$ & $0.95 \pm 0.06$ & $1.43 \pm 0.08$ & $<0.05$ \\
\hline VLDL-CHOL & $0.18 \pm 0.01$ & $0.22 \pm 0.03$ & NS & $10.11 \pm 0.18$ & $11.14 \pm 0.42$ & NS \\
\hline \multicolumn{7}{|c|}{ Triglyceride (mmol/L) } \\
\hline HDL-TG & $0.030 \pm 0.005$ & $0.044 \pm 0.003$ & $<0.05$ & $0.266 \pm 0.028$ & $0.293 \pm 0.024$ & NS \\
\hline LDL-TG & $0.095 \pm 0.012$ & $0.075 \pm 0.003$ & NS & $1.318 \pm 0.057$ & $1.302 \pm 0.220$ & NS \\
\hline VLDL-TG & $0.305 \pm 0.018$ & $0.390 \pm 0.048$ & NS & $64.648 \pm 1.841$ & $63.886 \pm 3.028$ & NS \\
\hline \multicolumn{7}{|c|}{ Phospholipid (mmol/L) } \\
\hline HDL-PL & $2.23 \pm 0.10$ & $3.55 \pm 0.10$ & $<0.0001$ & $1.02 \pm 0.04$ & $2.17 \pm 0.10$ & $<0.01$ \\
\hline LDL-PL & $0.20 \pm 0.03$ & $0.32 \pm 0.04$ & $<0.05$ & $0.75 \pm 0.09$ & $1.30 \pm 0.06$ & $<0.05$ \\
\hline VLDL-PL & $0.28 \pm 0.06$ & $0.36 \pm 0.08$ & NS & $11.38 \pm 0.32$ & $12.11 \pm 0.44$ & NS \\
\hline
\end{tabular}

HDL, LDL, and VLDL fractions were obtained from $1.5 \mathrm{~mL}$ pooled plasma from 2-3 male mice by ultracentrifugation. Cholesterol (CHOL), triglyceride (TG), and phospholipid (PL) levels in each lipoprotein fraction were determined by biochemical assays. Values are expressed as the mean $\pm \mathrm{SE}(\mathrm{mmol} / \mathrm{L}, n=6-8$ in each group).

in both mouse groups (Fig. 2B), thus causing a shift in lipoprotein fractions from HDL-CHOL (normolipidemic state) to predominantly VLDL/LDL (hyperlipidemic state). VLDL- and LDL-CHOL levels were similar in the two groups (Fig. 2B), while our ultracentrifugation study detected higher LDL-CHOL levels in EL-/- mice (Table 1). HDL-CHOL was significantly reduced by $\mathrm{P}-407$ in both groups, while EL-/- mice still showed an $80 \%$ higher HDL-CHOL level than WT mice (Fig. 2B).

Most TG was found in the VLDL fraction at baseline (Fig. 2C), without any significant difference in VLDL-TG between WT and EL-/- mice. When mice were treated with P-407, VLDL-TG markedly increased to $20-25 \mathrm{mmol} / \mathrm{L}$ in both groups, with similar levels for the groups (Fig. 2D). HDL-TG was increased after P-407 administration without any difference between WT and EL-/- mice. These results indicated that P-407 increased the TG content and decreased the CHOL content in HDL fractions, while EL inactivation mainly enhanced the CHOL content of HDL particles. No chylomicrons were detected in these fasted mice, supporting the notion that the increase in plasma TG was not reflected in chylomicron-TG levels.

HDL particle size analysis using HPLC found that HDL particles were larger in $E L-/-$ mice than in WT under non-stimulated conditions (Fig. 2E), suggesting that EL promotes the remodeling of HDL particles. P-407 administration resulted in an increase in HDL particle size in both WT and $E L-/-$ mice. Interestingly, the HDL particle size in $E L-/-$ mice was still larger than that in WT mice after P-407 treatment.

\section{Plasma TG-Lipase Activity is Inhibited by P-407}

We measured the activities of two major plasma TG-lipases, LPL and HL, in post-heparin plasma and found that they were similar in both WT and $E L-/-$ mice at baseline (Fig.3A). P-407 treatment markedly reduced TG-lipase activity in both groups by approximately 95\%, consistent with previous reports ${ }^{22}$. Notably, there was no difference in TG-lipase activity between groups.

Measurement of plasma phospholipase activity demonstrated that plasma phospholipase activity in $E L-/$ - mice was $40 \%$ lower than in WT mice at baseline. Following P-407 administration, plasma phospholipase activity decreased in WT $(p<0.05)$ but not in $E L-/-$ mice, while there was no difference in phospholipase activity between mouse groups (Fig.3B). These findings suggested that EL activity in vivo was inhibited by P-407 administration.

\section{P-407 does not Reduce Lipase Expression}

EL is predominantly expressed by vascular tissues, while HL is expressed in the liver, and LPL in the heart, muscles, and liver. We evaluated mRNA expression of the lipases in these different tissues. RNase protection assays disclosed that LPL, HL and EL levels in the liver were not affected by P-407 (Fig. 4A). Similarly, LPL expression in the heart was not changed by P-407 treatment (Fig. 4B). EL expression in the mouse aorta was evaluated by real-time 

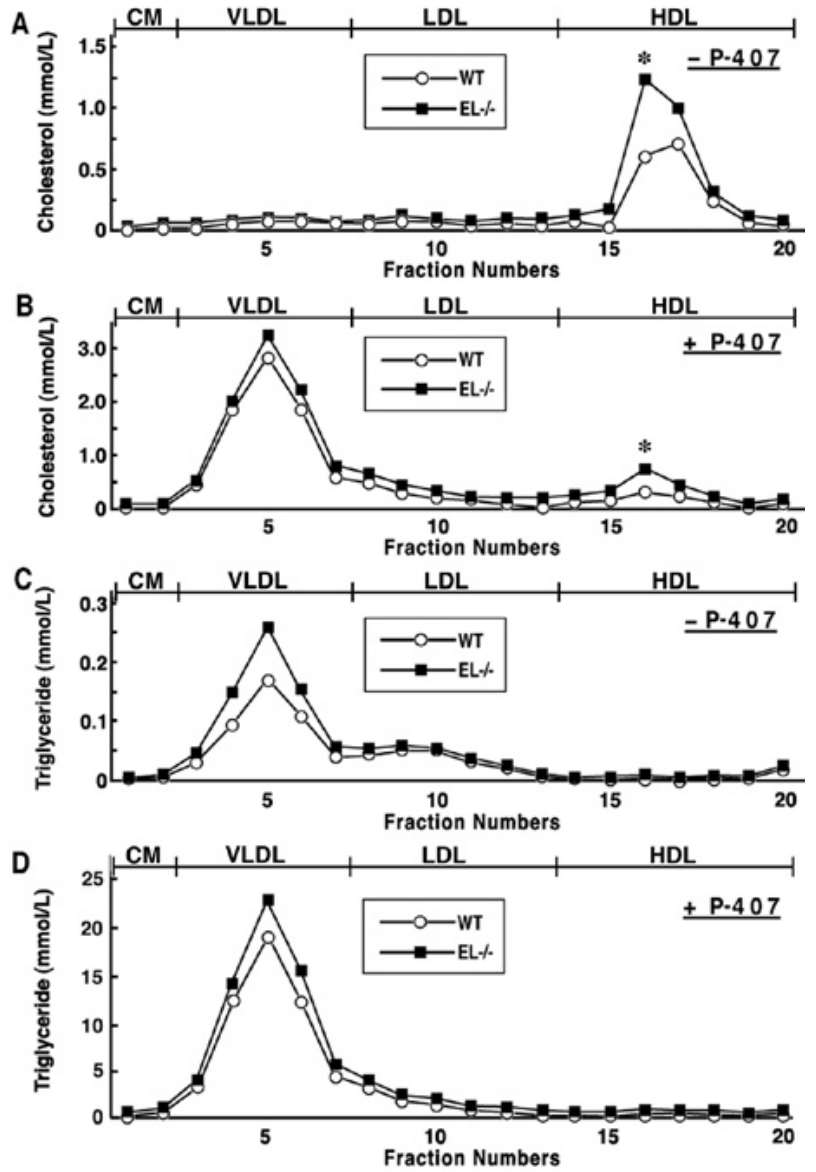

E

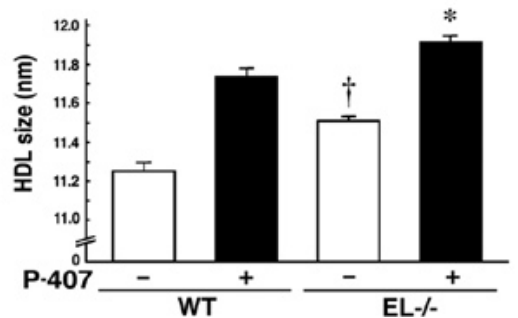

Fig. 2. Plasma lipoprotein profiles and HDL particle size after P-407 treatment.

Plasma samples were obtained from wild-type (WT) and endothelial lipase-deficient $(E L-/-)$ mice before and 16 hours after Poloxamer-407 (P-407, $0.5 \mathrm{~g} / \mathrm{kg}$, i.p.) injection, subjected to gel filtration chromatography, and cholesterol (CHOL) and triglyceride (TG) concentrations in each fraction were measured. Fractions 1-2 represent Chylomicron (CM), 3-7 VLDL, 8-13 LDL, and 14-20 HDL. A, CHOL concentration at baseline. B, CHOL concentration after P-407 injection. C, TG concentration at baseline. D, TG concentration after P-407 injection. E. High density lipoprotein (HDL) particle size was determined by gel filtration chromatography. Data are expressed as the mean \pm SE. ${ }^{*} p<0.05$ and ${ }^{\dagger} p<0.01$ vs. corresponding wild-type (WT) values ( $n=6-8$ in each group).

PCR because of the limited amount of RNA isolated, and was found to increase significantly in response to P-407 (Fig.4C). Western blotting revealed that EL
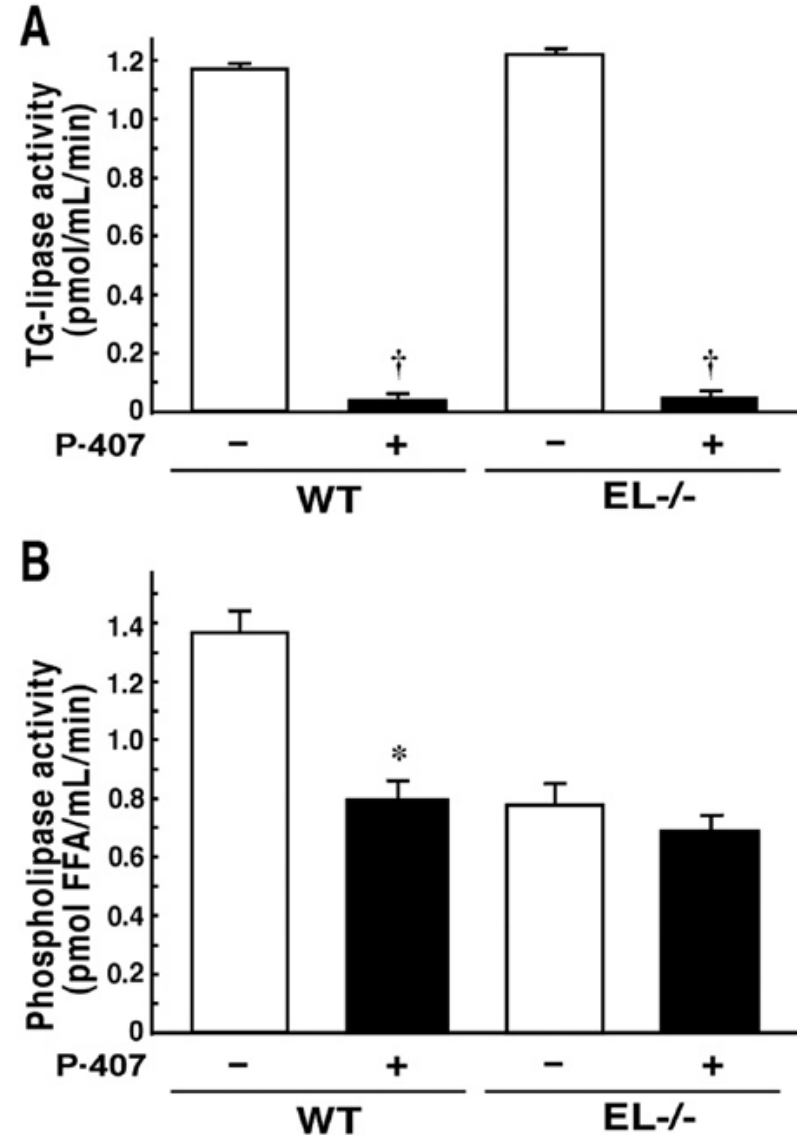

Fig. 3. Plasma lipase activity in P-407-treated mice.

Mice were treated with Poloxamer-407 (P-407), and plasma triglyceride (TG)-lipase activity (A) or phospholipase (PL) activity (B) was determined. ${ }^{*} p<0.05$ and ${ }^{\dagger} p<0.01$ vs. corresponding vehicle treatments ( $n=6-8$ in each group).

protein expression increased in the aorta but did not change in the liver (Fig. 4D). Despite the increase in EL expression in the aorta, EL protein levels in postheparin plasma were not significantly altered by $\mathrm{P}-407$ treatment (data not shown). These findings suggest that P-407 inhibits lipase activities without decreasing the mRNA levels of the lipases evaluated.

Next we examined to what extent EL expression was affected in vitro by $\mathrm{P}-407$ treatment. Human umbilical vein endothelial cells were stimulated with P-407 and EL mRNA was quantified with real-time PCR. The EL mRNA level increased in response to P-407 in a time- and dose- dependent manner (Fig. 5A, B), indicating that P-407 increases EL expression during the acute time course. To further investigate the effect of P-407 on EL enzymatic activity, we collected the culture medium of EL-overexpressing COS7 cells, and evaluated the phospholipase activities therein. As shown in Fig. 5C, P-407 significantly inhibited EL 

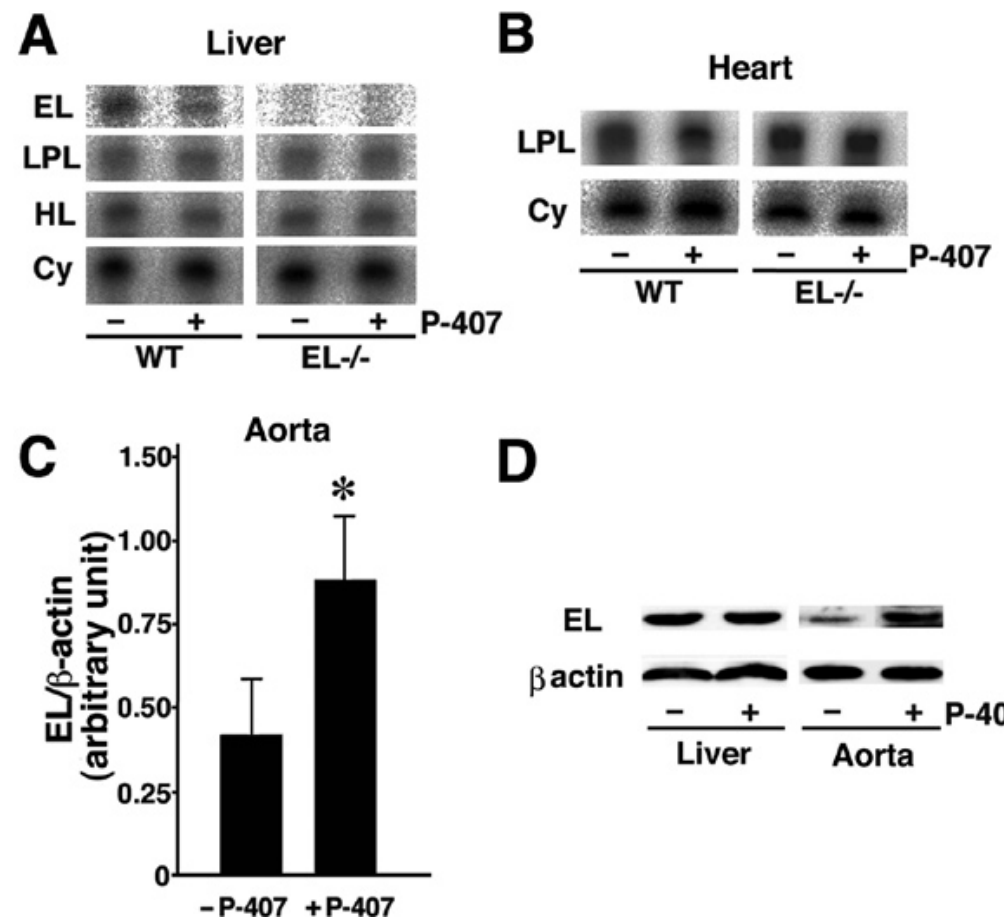

D

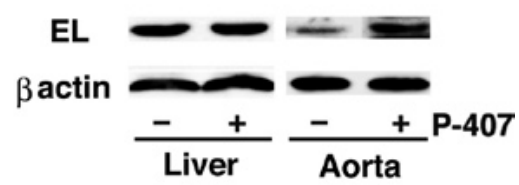

Fig.4. Expression of lipase members in P-407-treated mouse tissues.

A and B. Total RNA samples were extracted from the liver (A) and heart (B) of wild-type (WT) and endothelial lipase-deficient $(E L-/-)$ mice, and expressions of EL, lipoprotein lipase (LPL), and hepatic lipase (HL) were analyzed by RNase protection assays. Cy, cyclophilin. C. Expression of EL in mouse aorta was analyzed with real-time PCR, and standardized by $\beta$-actin mRNA level. ${ }^{*} p<0.05$ vs. vehicle $(n=4)$. D. Tissue lysate of the liver or aorta was subjected to Western blotting for EL expression. Representative images are shown in A, B, and D $(n=3-4)$.

phospholipase activity in vitro, indicating that P-407 eliminates the action of EL, but not by decreasing its expression.

\section{Reduction of HDL is Mediated by the Lipolytic and Bridging Function of EL}

It has been demonstrated that, besides lipolytic function, EL has a non-enzymatic bridging function between lipoproteins and the vascular wall ${ }^{19)}$. To determine the relative effects of the EL lipolytic function on HDL compared with the ligand-binding function, we injected the mice with AdEL (wild-type EL), AdEL-S149A (catalytically inactive mutant), and AdLacZ (control) from the tail vein. This procedure resulted in overexpression of the transgene only in the liver, while EL and EL-S149A protein were released into the plasma by treatment with heparin (Fig. 6A). AdEL injection resulted in a marked reduction in plasma HDL levels the next day, and this effect lasted for at least 28 days (Fig. 6B), as was also reported in previous papers ${ }^{8,19)}$. The injection of AdEL-S149A (Fig. 6B) or AdLacZ (data not shown) did not signifi- cantly affect plasma HDL levels under normolipidemic conditions in either mouse group.

Next, P-407-induced hyperlipidemia was generated in gene-transferred mice. When wild-type EL was overexpressed, plasma HDL levels were markedly reduced in both WT and $E L-/-$ mice (Fig. 6C). Interestingly, when EL-S149A was overexpressed, plasma HDL levels were significantly reduced in hypertriglyceridemic mice (Fig. 6C), but the treatment had no significant effect on normolipidemic mice (Fig. 6B). This result suggests that the low HDL levels in WT mice after P-407 treatment may be mediated through the lipolytic activity of EL as well as its ligand-binding function. AdEL injection resulted in a significant reduction of plasma TG levels in P-407-treated WT and $E L-/-$ mice, but AdEL-S149A injection did not (Fig. 6C).

To verify the molecular basis of the bridging function of EL in HDL metabolism, we finally examined the effects of AdEL-WT and AdEL-S149A on cellular uptake of DiI-labeled HDL. Confocal microscopy (Fig. 6D, top) or flow cytometry (Fig. 6D, bot- 
A

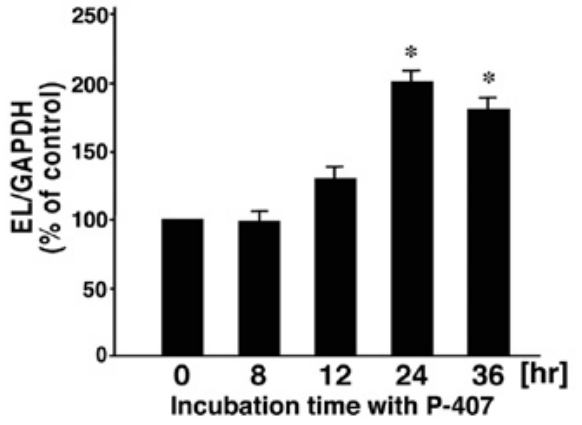

B

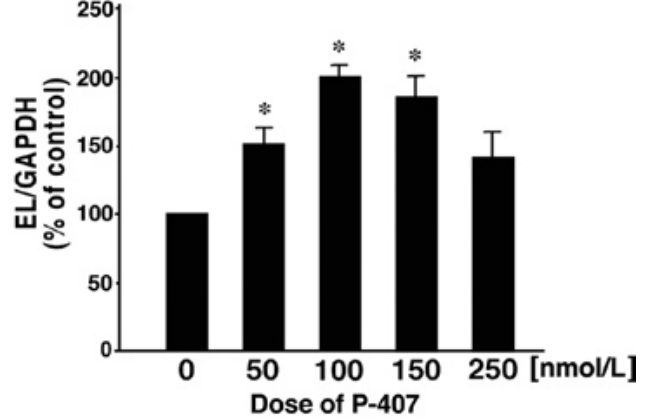

C

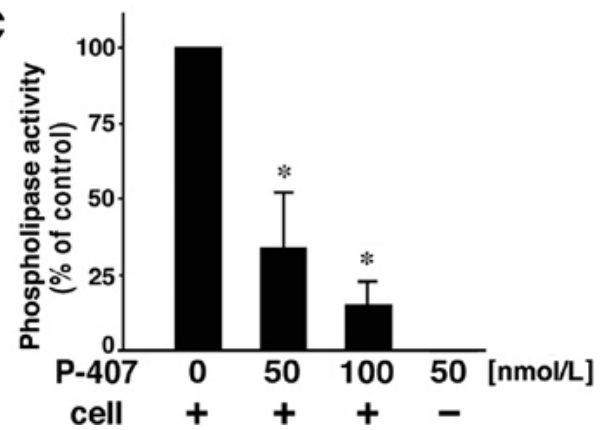

Fig. 5. Effects of P-407 on EL expression and activity in cultured endothelial cells.

A and B. Human umbilical vein endothelial cells (HUVEC) were stimulated with $100 \mathrm{nmol} / \mathrm{L}$ Poloxamer-407 (P-407) for the indicated time course (A), or with the indicated concentrations of P-407 for 24 hours (B). Expression of endothelial lipase (EL) was analyzed with real-time PCR, and standardized by GAPDH mRNA expression. ${ }^{*} p<0.05$ vs. vehicle control $(n=4)$. C. ELexpressing COS7 cells were incubated with P-407, and phospholipase activity in the concentrated culture medium was measured. The phospholipase activity of cell-free negative control and P-407free positive control was defined as $0 \%$ and $100 \%$, respectively. Data are expressed as a percentage of P-407-free positive control. $* p<0.05$ vs. vehicle control $(n=4)$.

tom) revealed that overexpression of EL-WT and EL-S149A resulted in enhanced uptake of HDL without significant differences in uptake between proteins. These findings support the notion that EL modulates plasma HDL levels through both its enzymatic and ligand-binding functions.

\section{Discussion}

Hypertriglyceridemia is the most common cause of low plasma HDL levels, and the mechanisms responsible for low HDL in hypertriglyceridemia have been previously studied. First, it was found that elevated plasma TG per se can lower HDL levels ${ }^{2,4)}$. An increase in plasma TG appears to promote the transfer of CHOL esters from HDL particles into TG-rich lipoproteins. This transfer is also promoted by CETP, which is the second cause of changes in HDL levels ${ }^{22)}$; however, mice are known to lack CETP activity in plasma. For our study, we confirmed that CETP activity was not detectable in WT and EL-/- mice before and after P-407 injection (data not shown). The third factor that correlates with HDL-CHOL levels is LCAT activity, which is necessary for the generation of CHOL esters for HDL particles. Although it has been reported that LCAT activity is impaired in EL-/mice ${ }^{23)}$, we found no difference in the endogenous CHOL esterification rate between WT and EL-/mice after P-407 treatment (data not shown). The fourth mechanism for low HDL levels in hypertriglyceridemia is the activity of TG-lipases, such as LPL or $\mathrm{HL}^{24,25)}$. When LPL-mediated lipolysis of TG-rich lipoproteins is blocked, HDL formation is disrupted ${ }^{26)}$, making it likely that $\mathrm{P}-407$ resulted in severe hypertriglyceridemia and defective HDL biosynthesis in our study. In P-407-induced hyperlipidemia, however, the activities of these lipases are almost completely inhibited to a similar extent in both mouse groups. Taken together, these findings suggest that the higher HDL level in EL-/- mice cannot be explained by changes in these known plasma factors. Regardless of whether mice were treated with P-407, EL-/- mice showed markedly higher HDL-CHOL levels than WT mice. EL may thus be a determinant of plasma HDL levels not only in the normolipidemic but also in the hypertriglyceridemic state induced by the administration of P-407.

It is believed that the effect of P-407 injection on plasma lipoprotein metabolism is primarily caused by the inhibition of plasma LPL and HL activity ${ }^{27,28)}$. In addition, P-407 was found to reduce the activity of cholesterol $7 \alpha$-hydroxylase ${ }^{12,29)}$. As a result, and concomitant with defective HDL biosynthesis, catabolism of TG-rich lipoproteins was delayed and plasma TG was increased markedly. Moreover, P-407 treatment increased the size and TG content of HDL particles, and TG-rich HDL particles are unstable because of the dissociation of apo A-I and are rapidly degraded by EL and $\mathrm{HL}^{30}$. These qualitative changes in $\mathrm{HDL}$ composition induced by $\mathrm{P}-407$ may accelerate HDL 
A
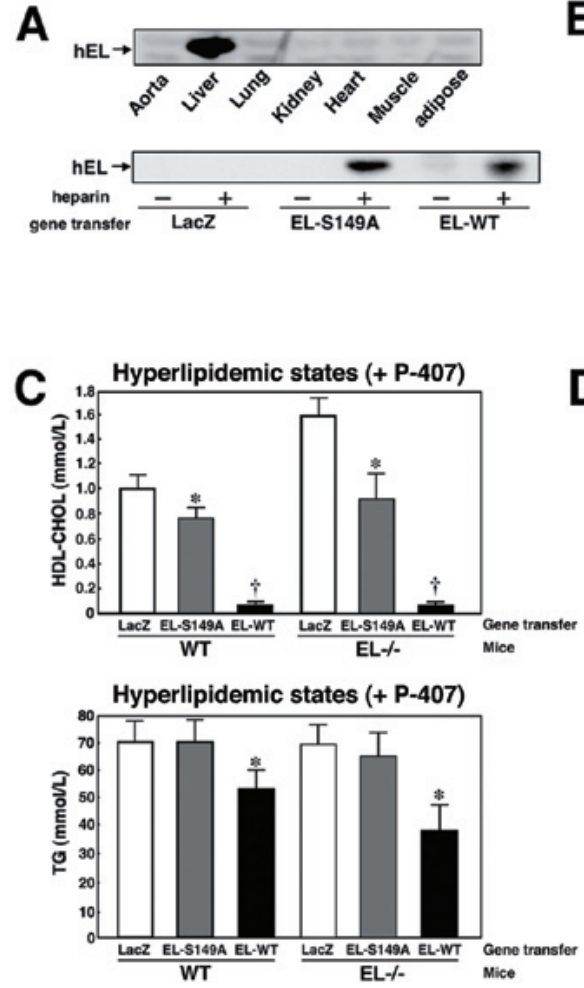

B

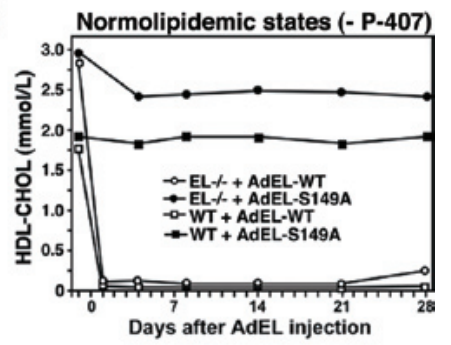

D
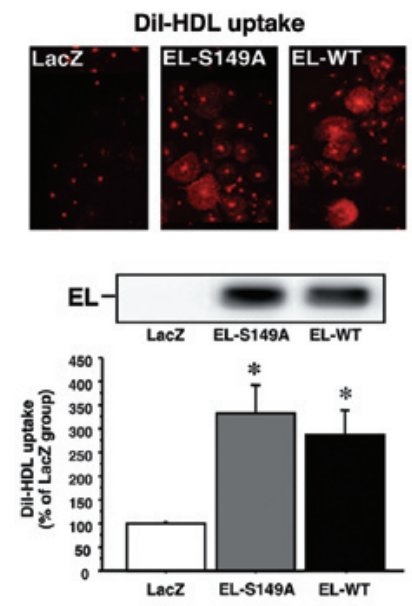

Fig. 6. Effects of EL overexpression on plasma lipids in P-407-treated mice.

WT and $E L-/-$ mice were injected with recombinant adenovirus encoding wildtype human EL (EL-WT), catalytically inactive human EL (EL-S149A) or LacZ (control). A, Western blotting for human EL expression in wild-type (WT) mice. B, Effect of EL overexpression on plasma HDL levels in normolipidemic states (without P-407). HDL-CHOL in EL-/- and WT mice markedly decreased by AdEL injection, while AdEL-S149A injection did not affect HDL-CHOL levels in these mice. C, Effects of EL gene transfer on plasma HDL-CHOL and TG in P-407-induced hypertriglyceridemic states. Overexpression of EL-WT resulted in a marked reduction of plasma HDL-CHOL levels both in WT and $E L-/-$ mice, while EL-S149A moderately reduced HDL-CHOL levels (top). Overexpression of EL-WT modestly reduced plasma TG levels both in WT and EL-/- mice, while EL-S149A did not (bottom panel). D, COS7 cells were infected with AdLacZ, AdEL-WT, or AdELS149A, and cellular uptake of DiI-labeled HDL was evaluated by confocal microscopy (top) or flowcytometry (bottom). The culture medium was subjected to Western blotting for standardization of protein expression (middle). ${ }^{*} p<0.05$ and ${ }^{\dagger} p<$ 0.01 vs. corresponding AdLacZ infection ( $n=6-8$ in each group).

catabolism in the model used in our study. Maugeais, et al. demonstrated that EL expression in mice resulted in a dose-dependent increase in plasma phospholipase activity, a fractional catabolic rate of HDL-apolipoprotein, and uptake of apoA-I in the kidney and liver ${ }^{31)}$. The increased HDL levels in P-407-treated $E L-/-$ mice are therefore likely to be the result not only of increased HDL levels at the time of injection but also of a decrease in the catabolic rate of HDL in EL-/- mice.

In this study, we have shown that P-407 inhibits EL activity both in vivo and in vitro. Our results show that phospholipase activity is similar in P-407-treated WT mice and $E L-/-$ mice, although there was a substantial level of residual phospholipase activity in the plasma. Because the phospholipase assay used in this study is not specific to EL phospholipase activity, it was speculated that residual phospholipase activity in these mice may reflect the activities of other phospholipases in plasma (Fig. 3B). If P-407 inhibits EL activity, however, both WT mice treated with P-407 and EL-/- mice are not supposed to possess active EL, so the difference in HDL-CHOL levels between P-407administered WT mice and EL-I- mice cannot be 
explained only by the phospholipase activity of EL. Since $E L-/-$ mice have undergone genetic manipulation of the $L I P G$ gene, they do not express EL. In contrast, P-407-treated WT mice do express EL, but it is catalytically inactive. In this context, EL has been shown to act as a molecular bridge between ELexpressing cells and lipoproteins, and mediates the binding and uptake of HDL holoparticles and selective uptake of HDL-associated cholesterol esters independent of its enzymatic activity ${ }^{32,33)}$. We therefore speculate that the presence of EL protein in P-407treated WT mice, even if catalytically inactive, may, through its bridging function, promote the clearance of HDL from the plasma, and partly contribute to the low HDL levels in WT mice. Our EL gene transfer data lent credence to this assumption. When wildtype EL was overexpressed in P-407-treated mice, HDL-CHOL levels diminished to $<0.1 \mathrm{mmol} / \mathrm{L}$, suggesting that P-407 treatment did not completely inhibit exogenously produced excess EL activity. Interestingly, EL-S149A overexpression resulted in a marked reduction of HDL-CHOL levels, not in normolipidemia but in hypertriglyceridemia, although the differences between these conditions leading to these different results remains a matter of conjecture. It has been reported that the expression of EL-S149A did not reduce the HDL-CHOL level in WT and apoA-I transgenic mice but resulted in a moderate reduction of HDL levels in HL-deficient mice ${ }^{19)}$. This suggests that the ligand-binding function of EL modulates HDL metabolism when HL is inactive. Similarly, EL has been shown to provide an alternative pathway for free fatty acid uptake in LPL-deficient tissue. Kratky, et al. demonstrated that EL is upregulated where LPL activity is absent, and that EL activity may contribute to the tissue uptake of free fatty acids, and thus affect the metabolism of plasma lipoproteins ${ }^{34)}$. In the light of previous findings, we speculate that EL may modulate plasma HDL levels through its lipolytic and ligand-binding functions in the hypertriglyceridemic state where the activity of TG-lipases is inhibited. Because our study focused on the role of EL in acute hypertriglyceridemia, further studies are required to establish the role of EL in HDL metabolism associated with chronic and more physiologic hypertriglyceridemia.

A number of previous studies have suggested that EL is a pro-inflammatory and pro-atherogenic molecule. Qiu, et al. indicated that suppression of EL expression attenuates proinflammatory cytokine secretion and influences the lipid composition in macrophages ${ }^{35)}$. Moreover, EL expression has been found to be associated with inflammation in patients with met- abolic syndrome with insulin resistance ${ }^{11)}$. EL expression has been reported to increase in mouse models of insulin resistance ${ }^{36)}$. In fact, EL deficiency reduced atherosclerosis in apolipoprotein-E-deficient mice ${ }^{16)}$. On the other hand, several studies have reported that EL may also have anti-inflammatory and anti-atherogenic effects. Ahmed, et al. demonstrated that HDL hydrolyzed by EL can activate $\operatorname{PPAR} \alpha$, resulting in attenuated leukocyte adhesion ${ }^{37)}$. In addition, it was found that EL can hydrolyze not only HDL but also apoB-containing lipoproteins and promote the catabolism of apoB-containing lipoproteins ${ }^{21)}$. Further studies are thus needed to clarify the overall effect of EL inactivation on atherosclerosis.

To summarize, EL was shown to play a role in HDL metabolism in mice in the normolipidemic as well as the acute hypertriglyceridemic state. EL can modulate plasma HDL-CHOL levels through both its lipolytic and ligand-binding functions in hypertriglyceridemic mice, yet the lipolytic activity of EL appears to be the main determinant of its effects on HDL metabolism in normolipidemic mice. Targeted inactivation of EL was found to result in the inhibition of hydrolysis and catabolism of HDL, and can increase plasma HDL levels when HDL biosynthesis is disrupted. Thus, EL may represent a novel target for HDL-raising pharmaceutical interventions for patients with low HDL levels.

\section{Acknowledgements}

This study was supported by Grants-In-Aid for Scientific Research, Global and 21st Century COE Program from the Ministry of Education, Culture, Sports, Science and Technology of Japan, a Sakakibara Memorial Research Grant from the Japan Research Promotion Society for Cardiovascular Diseases, Hyogo Foundation for Science and Technology, Meiji Yasuda Life Foundation of Health and Welfare, and Suzuken Memorial Foundation.

\section{References}

1) Cameron AJ, Shaw JE, Zimmet PZ: The metabolic syndrome: prevalence in worldwide populations. Endocrinol Metab Clin North Am, 2004; 33: 351-375

2) Davis CE, Gordon D, LaRosa J, Wood PD, Halperin M: Correlations of plasma high-density lipoprotein cholesterol levels with other plasma lipid and lipoprotein concentrations. Circulation, 1980; 62: IV24-30

3) Ginsberg HN: Lipoprotein physiology in nondiabetic and diabetic states. Relationship to atherogenesis. Diabetes Care, 1991; 14: 839-855

4) Nikkilä EA, Taskinen MR, Sane T: Plasma high-density 
lipoprotein concentration and subfraction distribution in relation to triglyceride metabolism. Am Heart J, 1987; 113: 543-548

5) Tato F, Vega GL, Grundy SM: Determinants of plasma HDL-cholesterol in hypertriglyceridemic patients. Role of cholesterol-ester transfer protein and lecithin cholesteryl acyl transferase. Arterioscler Thromb Vasc Biol, 1997; 17: 56-63

6) Heller DA, de Faire U, Pedersen NL, Dahlén G, McClearn GE: Genetic and environmental influences on serum lipid levels in twins. N Engl J Med, 1993; 328: 1150-1156

7) Hirata K, Dichek HL, Cioffi JA, Choi SY, Leeper NJ, Quintana L, Kronmal GS, Cooper AD, Quertermous T: Cloning of a unique lipase from endothelial cells extends the lipase gene family. J Biol Chem, 1999; 274: 1417014175

8) Jaye M, Lynch KJ, Krawiec J, Marchadier D, Maugeais C, Doan K, South V, Amin D, Perrone M, Rader DJ: A novel endothelial-derived lipase that modulates HDL metabolism. Nat Genet, 1999; 21: 424-428

9) Ishida T, Choi S, Kundu RK, Hirata K, Rubin EM, Cooper AD, Quertermous T: Endothelial lipase is a major determinant of HDL level. J Clin Invest, 2003; 111: 347355

10) Ma K, Cilingiroglu M, Otvos JD, Ballantyne CM, Marian AJ, Chan L: Endothelial lipase is a major genetic determinant for high-density lipoprotein concentration, structure, and metabolism. Proc Natl Acad Sci USA, 2003; 100: 2748-2753

11) Badellino KO, Wolfe ML, Reilly MP, Rader DJ: Endothelial lipase concentrations are increased in metabolic syndrome and associated with coronary atherosclerosis. PLoS Med, 2006; 3: e22

12) Johnston TP: The P-407-induced murine model of dosecontrolled hyperlipidemia and atherosclerosis: a review of findings to date. J Cardiovasc Pharmacol, 2004; 43: 595-606

13) Wout ZG, Pec EA, Maggiore JA, Williams RH, Palicharla P, Johnston TP: Poloxamer 407-mediated changes in plasma cholesterol and triglycerides following intraperitoneal injection to rats. J Parenter Sci Technol, 1992; 46: 192-200

14) Palmer WK, Emeson EE, Johnston TP: The poloxamer 407-induced hyperlipidemic atherogenic animal model. Med Sci Sports Exerc, 1997; 29: 1416-1421

15) Bronzert TJ, Brewer HB Jr: New micromethod for measuring cholesterol in plasma lipoprotein fractions. Clin Chem, 1977; 23: 2089-2098

16) Ishida T, Choi SY, Kundu RK, Spin J, Yamashita T, Hirata K, Kojima Y, Yokoyama M, Cooper AD, Quertermous T: Endothelial lipase modulates susceptibility to atherosclerosis in apolipoprotein-E-deficient mice. J Biol Chem, 2004; 279: 45085-45092

17) Usui S, Hara Y, Hosaki S, Okazaki M: A new on-line dual enzymatic method for simultaneous quantification of cholesterol and triglycerides in lipoproteins by HPLC. J Lipid Res, 2002; 43: 805-814

18) Usui $S$, Nakamura M, Jitsukata K, Nara M, Hosaki S, Okazaki M: Assessment of between-instrument variations in a HPLC method for serum lipoproteins and its trace- ability to reference methods for total cholesterol and HDL-cholesterol. Clin Chem, 2000; 46: 63-72

19) Broedl UC, Maugeais C, Marchadier D, Glick JM, Rader DJ: Effects of nonlipolytic ligand function of endothelial lipase on high density lipoprotein metabolism in vivo. J Biol Chem, 2003; 278: 40688-40693

20) Ishida $T$, Hirata $K$, Sakoda $T$, Kawashima $S$, Akita $H$, Yokoyama M: Identification of mRNA for 5-HT1 and 5-HT2 receptor subtypes in human coronary arteries. Cardiovasc Res, 1999; 41: 267-274

21) McCoy MG, Sun GS, Marchadier D, Maugeais C, Glick JM, Rader DJ: Characterization of the lipolytic activity of endothelial lipase. J Lipid Res, 2002; 43: 921-929

22) Mann CJ, Yen FT, Grant AM, Bihain BE: Mechanism of plasma cholesteryl ester transfer in hypertriglyceridemia. J Clin Invest, 1991; 88: 2059-2066

23) Ma K, Forte T, Otvos JD, Chan L: Differential additive effects of endothelial lipase and scavenger receptor-class B type I on high-density lipoprotein metabolism in knockout mouse models. Arterioscler Thromb Vasc Biol, 2005; 25: $149-154$

24) Blades B, Vega GL, Grundy SM: Activities of lipoprotein lipase and hepatic triglyceride lipase in postheparin plasma of patients with low concentrations of HDL cholesterol. Arterioscler Thromb, 1993; 13: 1227-1235

25) Goldberg IJ, Blaner WS, Vanni TM, Moukides M, Ramakrishnan R: Role of lipoprotein lipase in the regulation of high density lipoprotein apolipoprotein metabolism. Studies in normal and lipoprotein lipase-inhibited monkeys. J Clin Invest, 1990; 86: 463-473

26) Weinstock PH, Bisgaier CL, Aalto-Setälä K, Radner H, Ramakrishnan R, Levak-Frank S, Essenburg AD, Zechner R, Breslow JL: Severe hypertriglyceridemia, reduced high density lipoprotein, and neonatal death in lipoprotein lipase knockout mice. Mild hypertriglyceridemia with impaired very low density lipoprotein clearance in heterozygotes. J Clin Invest, 1995; 96: 2555-2568

27) Johnston TP, Palmer WK: Mechanism of poloxamer $407-$ induced hypertriglyceridemia in the rat. Biochem Pharmacol, 1993; 46: 1037-1042

28) Johnston TP, Nguyen LB, Chu WA, Shefer S: Potency of select statin drugs in a new mouse model of hyperlipidemia and atherosclerosis. Int J Pharm, 2001; 229: 75-86

29) Wasan KM, Subramanian R, Kwong M, Goldberg IJ, Wright T, Johnston TP: Poloxamer 407-mediated alterations in the activities of enzymes regulating lipid metabolism in rats. J Pharm Pharm Sci, 2003; 6: 189-197

30) Rashid S, Watanabe T, Sakaue T, Lewis GF: Mechanisms of HDL lowering in insulin resistant, hypertriglyceridemic states: the combined effect of HDL triglyceride enrichment and elevated hepatic lipase activity. Clin Biochem, 2003; 36: 421-429

31) Maugeais C, Tietge UJ, Broedl UC, Marchadier D, Cain W, McCoy MG, Lund-Katz S, Glick JM, Rader DJ: Dose-dependent acceleration of high-density lipoprotein catabolism by endothelial lipase. Circulation, 2003; 108: 2121-2126

32) Fuki IV, Blanchard N, Jin W, Marchadier DH, Millar JS, Glick JM, Rader DJ: Endogenously produced endothelial lipase enhances binding and cellular processing of plasma 
lipoproteins via heparan sulfate proteoglycan-mediated pathway. J Biol Chem, 2003; 278: 34331-34338

33) Strauss JG, Zimmermann R, Hrzenjak A, Zhou Y, Kratky D, Levak-Frank S, Kostner GM, Zechner R, Frank S: Endothelial cell-derived lipase mediates uptake and binding of high-density lipoprotein (HDL) particles and the selective uptake of HDL-associated cholesterol esters independent of its enzymic activity. Biochem J, 2002; 368: 69-79

34) Kratky D, Zimmermann R, Wagner EM, Strauss JG, Jin W, Kostner GM, Haemmerle G, Rader DJ, Zechner R: Endothelial lipase provides an alternative pathway for FFA uptake in lipoprotein lipase-deficient mouse adipose tissue. J Clin Invest, 2005; 115: 161-167

35) Qiu G, Ho AC, Yu W, Hill JS: Suppression of endothelial or lipoprotein lipase in THP-1 macrophages attenuates proinflammatory cytokine secretion. J Lipid Res, 2007; 48: 385-394

36) Yasuda T, Ishida T, Kojima Y, Tanaka H, Okada T, Fukuda A, Hara T, Inoue M, Hirata K: Overexpression of endothelial lipase in the liver promotes the clearance of plasma lipids but accelerates liver steatosis in a mouse model of metabolic syndrome. Circulation, 2007; 116: II-240

37) Ahmed W, Orasanu G, Nehra V, Asatryan L, Rader DJ, Ziouzenkova O, Plutzky J: High-density lipoprotein hydrolysis by endothelial lipase activates PPARalpha: a candidate mechanism for high-density lipoprotein-mediated repression of leukocyte adhesion. Circ Res, 2006; 98: 490-498 